\title{
ON ASYMPTOTIC DECOMPOSITIONS OF 0 -SOLUTIONS IN THE THEORY OF QUASILINEAR SYSTEMS OF DIFFERENCE EQUATIONS
}

A. V. Kostin and I. V. Skripnik

UDC 517.949

\begin{abstract}
We consider a quasilinear system of difference equations with certain conditions. We prove that there exists a formal partial o-solution of this system in the form of functional series of special type. We also prove a theorem on the asymptotic behavior of this solution.
\end{abstract}

Consider the system of difference equations

$$
\begin{gathered}
\Delta y_{k}(t)=q_{k}(t)+\sum_{i=1}^{n} p_{k i}(t) y_{i}(t)+\sum_{k_{i}+\ldots+k_{n}=2}^{\infty} p_{k k_{1} \ldots k_{n}}(t) y_{1}^{k_{1}}(t) \ldots y_{n}^{k_{n}}(t), \\
t \in N, \quad t \geq t_{0}, \quad k=1, \ldots, n,
\end{gathered}
$$

with the conditions

(i) $\left|p_{k k_{1} \ldots k_{n}}(t)\right| \leq A R^{-\left(k_{1}+\ldots+k_{n}\right)}$,

$$
k=1, \ldots, n, \quad k_{1}+\ldots+k_{n} \geq 2, \quad A, R \in \mathbb{R}_{+}
$$

(ii) $q_{k}(t)=o(1), \quad k=1, \ldots, n, \quad t \rightarrow+\infty$;

(iii) $\exists P_{0}=\lim _{t \rightarrow+\infty} P(t), \quad P_{0} \in \mathbb{C}^{n \times n}, \quad P(t)=\left(p_{k i}(t)\right)_{n}^{n}$.

Assume that the characteristic numbers $\lambda_{k}, k=1, \ldots, n$, of the matrix $P_{0}$ possess the property $\left|1+\lambda_{k}\right| \neq 0$, $k=1, \ldots, n$.

Inequality (2) guarantees the absolute and uniform convergence of the series in system (1) in any domain of the form

$$
\Gamma\left\{t \in N, t \geq t_{0}, \sum_{i=1}^{n}\left|y_{i}(t)\right| \leq R_{0}<R\right\}, \quad R_{0} \in \mathbb{R}_{+} .
$$

Furthermore, we assume that the functions $q_{k}(t), p_{k i}(t)$, and $p_{k k_{1} \ldots k_{n}}, k, i=1, \ldots, n, k_{1}+\ldots+k_{n} \geq 2$, admit, in a certain sense (see Definitions 6 and 7), formal expansions into series of the form

$$
\sum_{k_{1}+\ldots+k_{p}=0}^{\infty} c_{k_{1} k_{2} \ldots k_{p}} f_{1}^{k_{1}}(t) f_{2}^{k_{2}}(t) \ldots f_{p}^{k_{p}}(t), \quad c_{k_{1} k_{2} \ldots k_{p}} \in \mathbb{C},
$$

Odessa University, Odessa. Translated from Ukrainskii Matematicheskii Zhurnal, Vol. 49, No. 5, pp. 672-677, May, 1997. Original articie submitted May 15, 1995. 
where $f_{k}(t), k=1, \ldots, p$, is a fixed set of functions such that

$$
\Delta^{i} f_{k}(t)=o(1), \quad \Delta^{0} f_{k}(t) \stackrel{\text { def }}{=} f_{k}(t), \quad k=1, \ldots, p, i=0,1,2, \ldots
$$

In what follows, we denote the set of functions $f_{k}(t), k=1, \ldots, p$, by $(f)$.

We rewrite system (1) as

$$
\Delta Y(t)=Q(t)+P(t) Y(t)+\Psi(t, Y(t))
$$

Consider the series

$$
\sum_{s=0}^{\infty} c_{k} \sigma_{k}(t), \quad \sigma_{k}(t)=\prod_{i=0}^{s-1}\left(\Delta^{i} f_{1}(t)\right)^{k_{i}} \ldots \prod_{i=0}^{s-1}\left(\Delta^{i} f_{p}(t)\right)^{l_{p}}
$$

where $k=k_{0} \ldots k_{s-1} \ldots l_{0} \ldots l_{s-1}$ and, for any fixed value $s$, the exponents $k_{0}, \ldots, k_{s-1}, \ldots, l_{0}, \ldots, l_{s-1}$ can be integer nonnegative numbers satisfying the condition

$$
k_{0}+2 k_{1}+\ldots+s k_{s-1}+\ldots+l_{0}+2 l_{1}+\ldots+s l_{s-1}=s
$$

The coefficients $c_{k}$ are columns of the same dimension $n$. The numbers $s$ are called the orders of the corresponding terms in (5).

Definition 1. A vector function $\varphi(t), t \in N, t \geq t_{0}$, which is a finite sum of the type

$$
\varphi(t)=\sum_{s=s_{0}} c_{k}^{*} \sigma_{k}(t), \quad c_{k}^{*} \in \mathbb{C}^{n \times 1}, \quad k=k_{0} \ldots k_{s-1} \ldots l_{0} \ldots l_{s-1}
$$

where the terms have the same order $s_{0}$, is called a function of order $s_{0}$, which is denoted as follows: $\Pi(\varphi(t))=s_{0}$.

Property 1. If $\Pi(\varphi(t))=s_{0}$ and $c \in \mathbb{C}$, then $\Pi(c \varphi(t))=s_{0}$.

Property 2. If $\Pi\left(\varphi_{1}(t)\right)=s_{0}$ and $\Pi\left(\varphi_{2}(t)\right)=s_{0}$, then

$$
\Pi\left(\varphi_{1}(t)+\varphi_{2}(t)\right)=s_{0}
$$

Property 3. If $\Pi\left(\varphi_{1}(t)\right)=s_{1}$ and $\Pi\left(\varphi_{2}(t)\right)=s_{2}$, then

$$
\Pi\left(\varphi_{1}(t) \varphi_{2}(t)\right)=s_{1}+s_{2}
$$

Definition 2. The following series are called, respectively, the sum, difference, and product of two formal series $\sum_{s=0}^{\infty} W_{s}$ and $\sum_{s=0}^{\infty} V_{s}$ of type (5):

$$
\sum_{s=0}^{\infty}\left(W_{s}+V_{s}\right), \quad \sum_{s=0}^{\infty}\left(W_{s}-V_{s}\right), \quad \sum_{s=0}^{\infty}\left(W_{s} V_{s}+W_{1} V_{s-1}+\ldots+W_{s} V_{0}\right)
$$

